\title{
Influence of Dispersant and ZDDP on Soot Wear
}

\author{
A. Kontou ${ }^{1}$ (1) $\cdot$ M. Southby ${ }^{2} \cdot$ N. Morgan ${ }^{2} \cdot$ H. A. Spikes ${ }^{1}$
}

Received: 3 August 2018 / Accepted: 24 October 2018 / Published online: 9 November 2018

(c) The Author(s) 2018

\begin{abstract}
Diesel engines and gasoline direct injection (GDI) engines both produce soot due to incomplete combustion of the fuel and some enters the lubricant where it accumulates between drain intervals, promoting wear of rubbing engine components. Currently the most favoured mechanism for this wear is that the anti-wear additives present in engine oils, primarily zinc dialkyldithiophosphates (ZDDPs), react very rapidly with rubbing surfaces to form relatively soft reaction products. These are easily abraded by soot, resulting in a corrosive-abrasive wear mechanism. This study has explored the impact of engine oil dispersant additives on this type of wear using combinations of dispersant, ZDDP and carbon black, a soot surrogate. It has been found that both the concentration and type of dispersant are critical in influencing wear. With most dispersants studied, wear becomes very high over an intermediate dispersant concentration range of $c a 0.1-0.4 \mathrm{wt} \% \mathrm{~N}$, with both lower and higher dispersant levels showing much less wear. However a few dispersants appear able to suppress high wear by ZDDP and carbon black over the whole concentration range. A series of experiments have been carried out to determine the origin of this behaviour and it is believed that high levels of dispersant, and, for a few dispersants, all concentration levels, protect the iron sulphide tribofilm initially formed by ZDDP from abrasion by carbon black.
\end{abstract}

Keywords Soot wear $\cdot$ ZDDP $\cdot$ Corrosive-abrasive wear $\cdot$ Dispersant $\cdot$ Succinimide $\cdot$ Carbon black

\section{Introduction}

High levels of soot in engine lubricants are frequently reported to induce high wear rates of engine components. Engine bearings, camshaft and crankshaft, piston rings, cylinder walls and timing chain are some of the engine parts that are most affected by wear induced by soot [1-3].

Many conflicting theories and mechanisms have been suggested as to how engine soot increases wear and how different additive combinations influence this during rubbing. Early work proposed that soot might adsorb anti-wear additives $[4,5]$ or compete with them for rubbing surfaces [6]. Other mechanisms suggested by different studies were that soot can induce enhanced oil degradation [7], metal reduction from anti-wear $\mathrm{Fe}_{3} \mathrm{O}_{4}$ to pro-wear $\mathrm{FeO}$ [8], lubricant

H. A. Spikes

h.spikes@imperial.ac.uk

1 Tribology Group, Department of Mechanical Engineering, Imperial College London, London, UK

2 Shell Centre, Shell Global Solutions Ltd, York Road, London SE1 7NA, UK starvation [9-11] and abrasion, either of the rubbing surfaces $[12,13]$ or anti-wear film $[4,12-21]$.

One mechanism to gain favour over the last few years is one in which soot abrades the relatively soft ferrous sulphide- and/or phosphate-based films initially formed by reaction of anti-wear additives with rubbed surfaces [19, 22], resulting in a corrosive-abrasive wear mechanism [23]. The main evidence for this is that when both zinc dialkyldithiophosphate (ZDDP) and the soot surrogate carbon black (CB) are present together in a lubricant, the combination can lead to much higher wear than if either ZDDP or CB is absent. Booth et al. studied additive combinations with CB using factorial analysis that showed that primary ZDDP appeared to increase pin wear [24]. They showed that blends containing both ZDDP and CB exhibited high wear on the ball, which they ascribed to "immature" anti-wear film formation. In 2010, Olomolehin et al. investigated the influence of $\mathrm{CB}$ and other nanoparticles on wear in anti-wear additivecontaining model lubricants [19]. They found that when $\mathrm{CB}$ was added to an oil containing ZDDP, the combination gave much more wear than when no ZDDP was present; i.e. ZDDP became pro-wear. They suggested a corrosiveabrasive mechanism in which the $\mathrm{CB}$ removes the initial 
iron sulphide and phosphate anti-wear film as rapidly as it forms, leading to a rapid loss of ferrous compounds and thus high wear levels. Salehi et al. have recently demonstrated similar high wear behaviour for CB in formulated engine oil [21] while Hu et al. have shown the contrary, with lower wear for formulated engine oil than one containing just dispersant and CB [25]. It has also been shown recently that high levels of soot wear occur in particular when rubbing steel surfaces are very hard, with much less wear with softer surfaces [26]. This is contrary to the dependence of wear on hardness expected for conventional abrasive or adhesive wear but accords with a chemically-mediated wear process where the initial ZDDP reaction rate depends on the asperity contact stress.

Although the evidence for a corrosive-abrasive wear mechanism is convincing, it is not yet clear whether this wear mechanism is universal or whether it can be mitigated by other additives present in the lubricant and in particular by the dispersant. This study aims to understand further the interaction of soot with lubricant additives and its impact on wear and to derive concrete conclusions and identify control measures.

\section{Experimental Materials}

A Gas to Liquid (GTL) base oil with viscosity $18.3 \mathrm{cSt}$ at $40{ }^{\circ} \mathrm{C}$ and $4.2 \mathrm{cSt}$ at $100{ }^{\circ} \mathrm{C}$ was used to formulate all lubricants. The process of obtaining engine soot from lubricants is time-consuming and the soot extracted will be contaminated by additives specific to the lubricant and fuel used. Soot can also vary in the degree of graphitization and particle size depending on engine conditions. Therefore, a soot surrogate such as furnace or channel carbon black (CB) is generally used for wear studies. In this study the CB Vulcan XC72R (Cabot UK Ltd.) was used as soot surrogate. Based on Transmission Electron Microscopy (TEM) it has a primary particle diameter of $20-50 \mathrm{~nm}$ and it can form secondary structures of about $400 \mathrm{~nm}$. Figure 1 shows a typical secondary particle made up of fused primary particles. In this study a concentration of $5 \mathrm{wt} \%$ of the CB was used unless stated otherwise.

Two types of lubricant additive were employed. Three ZDDPs were studied as listed in Table 1. For most tests 0.08 $\mathrm{wt} \% \mathrm{P}$ was employed unless stated otherwise. This level of $\mathrm{P}$ is the maximum limit allowed in most commercial passenger car engine oils, though higher levels may be present in heavy duty diesel engine oils.

To maintain soot in suspension, a dispersant is essential and as shown in Table 1, eight different dispersants were studied. All were polyisobutylene succinimide (PIBSA) polyamine dispersants. They were used at a wide range of dispersant concentrations, 0.002, 0.02, 0.05, 0.1, $0.2 \mathrm{wt} \%$

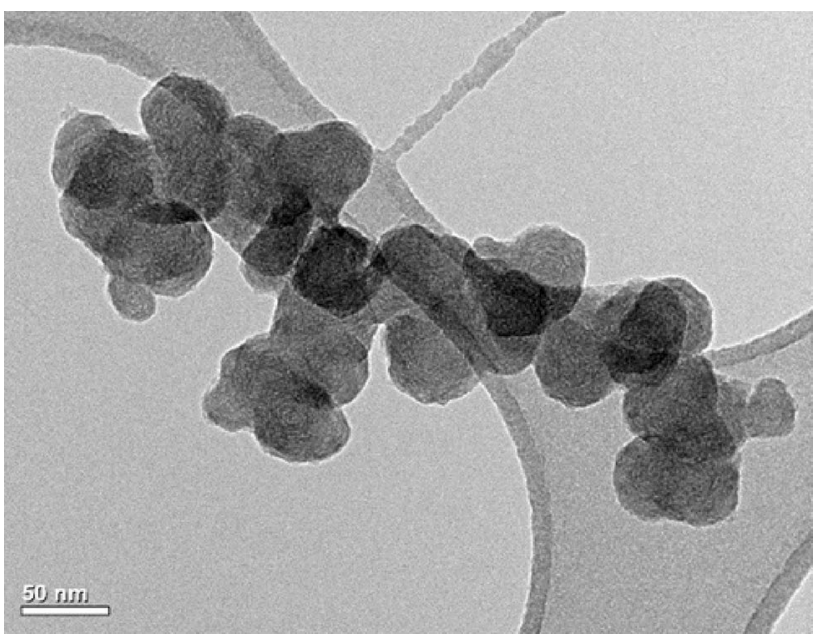

Fig. 1 TEM picture of primary particles of CB Vulcan XC72R forming a secondary structure

$\mathrm{N}$. In order to compare the various dispersants, these concentrations are described in this study in terms of $\mathrm{N} \mathrm{wt} \%$ rather than dispersant $w t \%$. The former focusses directly on the functionality of the dispersants (i.e. the number of available amine/imide groups per molecule). This is most likely to drive soot/surface interactions by adsorption on the CB surface, and is preferred to the overall dispersant wt $\%$ that reflects MWt. and thus PIB chain length. It was not possible to study $\mathrm{CB}$ in the absence of dispersant since it would not disperse at all; $0.002 \% \mathrm{~N}$ was the minimum that allowed a reasonably homogeneous mixture to be obtained.

The ZDDP/dispersant solutions were blended and stirred using a magnetic stirrer at $70{ }^{\circ} \mathrm{C}$ for $30-40$ min until the solution was fully homogeneous. Then $\mathrm{CB}$ that has been dried in an oven at $100{ }^{\circ} \mathrm{C}$ was added to the samples and stirred on the magnetic stirrer for $30 \mathrm{~min}$ at $70{ }^{\circ} \mathrm{C}$. The resulting dispersions were tested immediately. Wear tests were performed in a High Frequency Reciprocating ball on flat rig (HFRR) by PCS Instruments, Acton, UK.

\section{Experimental Methods}

\subsection{HFRR Wear Tests}

The HFRR is a controlled reciprocating friction and wear testing device employed to assess the performance of fuels and lubricants. The test uses a $6 \mathrm{~mm}$ diameter ball loaded and reciprocated against the flat surface of a stationary disc immersed in the lubricant. Table 2 shows the HFRR specimen properties. The HFRR test conditions are listed in Table 3. All the tests were repeated twice and error bars using standard error, $\sigma / \sqrt{ } \mathrm{n}$, were plotted for each average value where $\sigma$ is the standard deviation and $n$ is the 
Table 1 Additives studied

\begin{tabular}{ll}
\hline Additive abbrev. & Additive details \\
\hline ZDDP1 & Mixed primary/secondary zinc \\
ZDDP2 & Primary zinc \\
ZDDP3 & Secondary zinc \\
D1 & PIBSA polyamine dispersant (high MWt.) \\
D2 & PIBSA polyamine dispersant (no information) \\
D3 & PIBSA polyamine dispersant (high MWt./high functionality) \\
D4 & PIBSA polyamine dispersant (high MWt./medium functionality) \\
D5 & PIBSA polyamine dispersant (high MWt./medium functionality) \\
D6 & PIBSA polyamine dispersant (medium MWt./low functionality) \\
D7 & PIBSA polyamine dispersant (high MWt./high functionality) \\
D8 & PIBSA polyamine dispersant (very high MWt./high functionality) \\
\hline
\end{tabular}

Table 2 HFRR test specimen properties

\begin{tabular}{ll}
\hline Ball and disc material & $\begin{array}{l}\text { LESCALLOY® 52100 VAC- } \\
\text { ARC® high performance bearing } \\
\text { steel }\end{array}$ \\
\hline Ball hardness (HV) & $880 \pm 3$ \\
Disc hardness (HV) & $772 \pm 57$ \\
Ball roughness, Ra (nm) & $5.0 \pm 0.1$ \\
Disc roughness, $\mathrm{Ra}(\mathrm{nm})$ & $5.1 \pm 0.2$
\end{tabular}

Table 3 HFRR wear test conditions

\begin{tabular}{ll}
\hline Load $(\mathrm{N})$ & $3.92(0.4 \mathrm{~kg})$ \\
Maximum hertz contact pressure $(\mathrm{GPa})$ & 1.03 \\
Frequency $(\mathrm{Hz})$ & 50 \\
Stroke length $(\mathrm{mm})$ & 1 \\
Test temperature $\left({ }^{\circ} \mathrm{C}\right)$ & 100 \\
Test duration $(\mathrm{min})$ & 60 \\
\hline
\end{tabular}

number of repeats. 95\% confidence intervals were estimated for each pair of repeats using a t-distribution:

$\bar{x} \pm t_{n-1} \frac{\sigma}{\sqrt{n}}$

where $\bar{x}$ is the average value. All the average values fell within their estimated confidence intervals.

The mean sliding speed in the HFRR under the test conditions used was $0.1 \mathrm{~m} / \mathrm{s}$, so the mean entrainment speed was $0.05 \mathrm{~m} / \mathrm{s}$. Elastohydrodynamic film thickness measurements were made using optical interferometry on the soot-free test oils in pure rolling conditions at $100{ }^{\circ} \mathrm{C}$ and showed films of thickness $4 \mathrm{~nm}$ and $5 \mathrm{~nm}$ at this entrainment speed for base oil and dispersant solution respectively at $0.2 \mathrm{wt} \% \mathrm{~N}$. Allowing for the difference in ball radius between the HFRR ( $3 \mathrm{~mm}$ ) and EHD tests
$(8.5 \mathrm{~mm})$, this implies a mean lambda ratio of $c a .0 .2$ at the start of an HFRR test, indicative of boundary lubrication conditions for all test fluids.

\subsection{Scanning White Light Interferometer (SWLI) Wear Measurement}

At the end of each HFRR test, the ball and disc were removed from the test rig, rinsed with toluene and iso-propanol and then treated with a $0.05 \mathrm{wt} \%$ solution of ethylene diamine tetraacetic acid (EDTA) for $40 \mathrm{~s}$. The last of these was to remove any ZDDP anti-wear film on the surfaces since this can interfere with optically-based wear measurement. Topography images were then obtained and analysed to determine the wear volume removed from the ball and the disc using a scanning white light interferometer (SWLI) Veeco Wyko model NT9100. For this project the machine was set in the Vertical Scanning Interferometry (VSI) mode, calibrated to measure rough surfaces with a nanometre detection range.

\subsection{Rheology Tests}

The CB-containing test samples were tested using a Discovery HR-1 rheometer to obtain the rheological characteristics of the samples at different $\mathrm{N}$ concentrations. The tests were carried out using a conical geometry of $40 \mathrm{~mm}$ diameter and $0.5^{\circ}$ cone angle and a Peltier plate at a controlled temperature of $100{ }^{\circ} \mathrm{C}$, which is close to nominal engine operating conditions. Flow peak-hold viscosity measurements were recorded at shear rates of 0.1 up to $100 / \mathrm{s}$.

\subsection{Dynamic Light Scattering for Particle Size Measurements}

Dynamic Light Scattering (DLS) measures the diffusion of particles moving under Brownian motion and converts this to particle size and size distribution using the 
Stokes-Einstein relationship. A Zetasizer $\mu \mathrm{V}$ from Malvern Instruments Ltd was employed in this study to obtain z-average diameter sizes of $\mathrm{CB}$ particles in solutions of different dispersant concentrations. The high viscosity of the samples containing very low dispersant concentrations limits the movement of the particles, so for particle size measurement the samples were diluted with heptane at a ratio of $1.5 \mu \mathrm{l}$ of $\mathrm{CB}$ solution to $7.5 \mathrm{ml}$ of heptane. Ultra-sonication of the samples was performed for $10 \mathrm{~min}$ to mix the CB samples with heptane and break down the large aggregates that were initially formed in heptane thereby avoiding high polydispersity indices. The tests were performed at $25{ }^{\circ} \mathrm{C}$ and the viscosity of heptane at this temperature was $0.38 \mathrm{mPa} \mathrm{s}$. This sample preparation procedure was adopted from La Rocca et al. [27]. The data were analysed using the Zetasizer software 7.11 version.

\subsection{Rolling/Sliding MTM-SLIM Tests for Tribofilm Formation and Removal}

Minitraction Machine-Spacer Layer Imaging (MTM-SLIM) from PCS Instruments (Acton, UK) was used in this study to record the impact of soot and dispersant on film formation by ZDDP and its removal by CB. A $19 \mathrm{~mm}$ diameter AISI 52100 steel ball was loaded against the flat surface of a rotating AISI 52100 steel disc which was immersed in the oil sample. The test conditions were load $31 \mathrm{~N}$, entrainment speed $0.1 \mathrm{~m} / \mathrm{s}$, slide-roll ratio $50 \%$ and test temperature $100{ }^{\circ} \mathrm{C}$. To monitor film formation, the spacer layer imaging method (SLIM) was employed. This uses a camera and optical interferometry to measure the thickness of thin transparent films formed on the ball during the test.

Two different tests were carried out: (i) tribofilm formation tests to study the impact of different dispersants on ZDDP formation rate and (ii) tribofilm formation and its subsequent removal by $\mathrm{CB}$. For the latter, after the film formation using a ZDDP + dispersant solution, the solution was drained and replaced by a sample with the same ZDDP and dispersant but including $5 \mathrm{wt} \%$ of $\mathrm{CB}$.

\subsection{Reciprocating MTM to Study Initial ZDDP Film Formation}

The MTM was also used in reciprocating, pure sliding mode to study the influence of dispersants on initial ZDDP film formation [28]. A stationary MTM ball is loaded at $20 \mathrm{~N}$ against an MTM disc that rotates back and forth at $20 \mathrm{~Hz}$ over a stroke length of $4 \mathrm{~mm}$. Tests were conducted at $130{ }^{\circ} \mathrm{C}$ and lasted just $5 \mathrm{~s}$ of rubbing in order to study the very first stages of ZDDP tribofilm formation. At the end of each test the ball was rinsed with heptane and then the rubbed scar analysed using SEM-EDS as described below.

\subsection{SEM-EDS Surface Analysis}

An HITACHI S-3400N SEM (Scanning Electron Microscopy) was used to capture high resolution images of the wear scars and the tribofilm topography when ZDDP was used in the lubricant. Some imaging was also carried out using an LEO Gemini 1525 FEG-SEM (Field Emission Gun-SEM). This has higher resolution and was able to identify CB nanoparticles on the surface. EDS (energy-dispersive X-ray spectroscopy) from Oxford instruments X-ray System INCA, was employed to detect any ZDDP film on the surfaces and also to analyse the chemical properties of anti-wear films. For EDS analysis, the same square region was selected inside the wear scars for all the samples. An accelerating voltage of $15 \mathrm{keV}$ and a working distance of $10 \pm 1 \mathrm{~mm}$ were used.

\subsection{TEM-EDS Tribofilm Analysis Using FIB Milling Technique}

A Helios 600 NanoLab FIB instrument was used to prepare cross section samples of the wear scars for Transmission Electron Microscope (TEM) with EDS analysis to observe any possible films/reactions at the surface that are not visible by any other method. This instrument is a dual beam SEM/ FIB system equipped with a gallium ion source operating in a range of $0.5-30 \mathrm{kV}, 1.5 \mathrm{pA}$ to $21 \mathrm{nA}$ beam current and an Omniprobe micromanipulator for in-situ lift out the TEM lamella. The advantage of this instrument is that, because of the combination of SEM and FIB, the preparation of the TEM lamella using the ion beam under the electron beam can be viewed directly which allows the milling to be precisely monitored without damaging the sample.

In most cases, TEM sample preparation consisted of milling a thin slice of the material perpendicular to the sample surface. After attaching the lamella to the micromaniputator's needle, the sample is lifted out from the bulk and attached to a special TEM grid. Then, low-voltage focused ion-beams at grazing incidence are used to thin the sample. Prior to the milling, the sample was first covered with a layer of $60 \mathrm{~nm}$ of gold deposited at low energy and then a thick layer of platinum, to protect it from damaging it during the milling procedure.

The high resolution JEOL JEM 2100F TEM was used to probe the structural characteristics of the film. Chemical characterisation was carried out using an EDS Oxford Instruments X-ray System INCA. 
Fig. 2 HFRR ball wear volume results at various $\mathrm{N}$ concentrations of a D1, ZDDP and BO without $\mathrm{CB}$; b with $\mathrm{CB} ;(0.08$ wt \% P, 5 wt \% CB). (Color figure online)

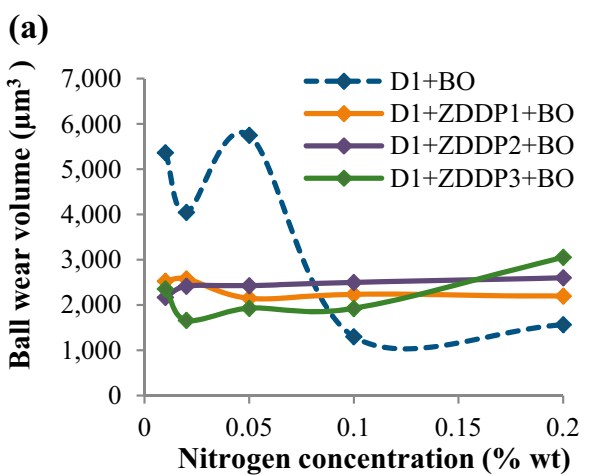

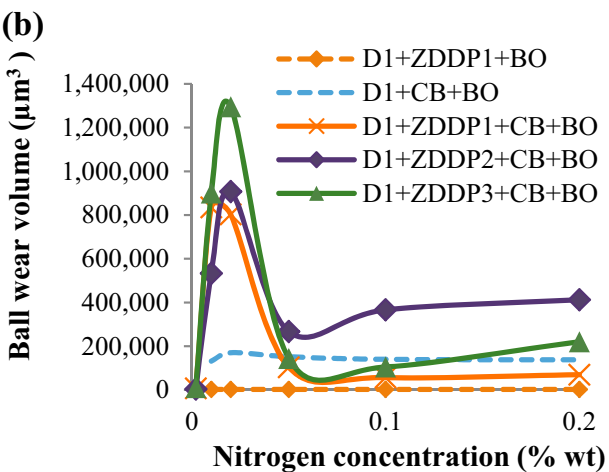

\section{HFRR Wear Test Results}

\subsection{Effect of Nitrogen Concentration}

Figure 2a shows the HFRR ball wear results for dispersant D1 alone and in combination with three ZDDPs: a mixed (ZDDP1), a primary (ZDDP2) and a secondary (ZDDP3) in base oil (BO). D1 solution in BO shows wear to be sensitive to $\mathrm{N}$ concentration, with wear reducing as $\mathrm{N}$ concentration increases. This indicates some weak boundary lubricating ability of the dispersant reducing the mild adhesive wear that occurs with base oil alone. However, when ZDPP is added, the wear volumes are almost independent of $\mathrm{N}$ content, indicating that the wear-controlling properties of ZDDP outweigh those of dispersant. Interestingly, ZDDP slightly increases wear when added to solutions of high dispersant concentration suggesting mild corrosive wear. All ZDDPs show similar wear results. As can be seen in Fig. 2a, the range of wear volumes of ZDDP and dispersant blends between 1500 and $3000 \mu \mathrm{m}^{3}$.
When $\mathrm{CB}$ is added to the same oil blends, wear volumes increase by a factor of up to $c a 500$, as shown in Fig. $2 \mathrm{~b}$. Some of this increase is seen with just dispersant and CB as indicated by the dashed blue line in Fig. 2b. This is largely independent of dispersant concentration and probably arises from abrasive wear of iron oxides by the $\mathrm{CB}$. However when both ZDDP and CB are present the trend of wear is strikingly different, with a maximum at $0.02 \mathrm{wt} \% \mathrm{~N}$ and much lower wear at higher, $(0.05 \mathrm{wt} \% \mathrm{~N})$, and lower, $(0.002 \mathrm{wt} \%$ $\mathrm{N})$, dispersant concentrations. The ZDDP has become prowear instead of anti-wear over much of the dispersant concentration range.

Since ZDDP3 gives the highest wear at $0.02 \mathrm{wt} \% \mathrm{~N}$ it was chosen to be further tested with eight dispersants of various molecular weights and functionalities. The results are shown in Fig. 3, again as a function of $\mathrm{N}$ concentration. Most dispersants give a similar general pattern of wear response to that shown in Fig. 2b, with a maximum wear at $0.02 \mathrm{wt} \% \mathrm{~N}$. However, a few (D3, D8 and to a lesser extent D7) show a strikingly different response, with low wear volumes at all concentrations.
Fig. 3 HFRR ball wear volume of fluids containing ZDDP3 and eight different dispersants at various $\mathrm{N}$ concentrations; $(0.08$ wt\% P, 5 wt\% CB)

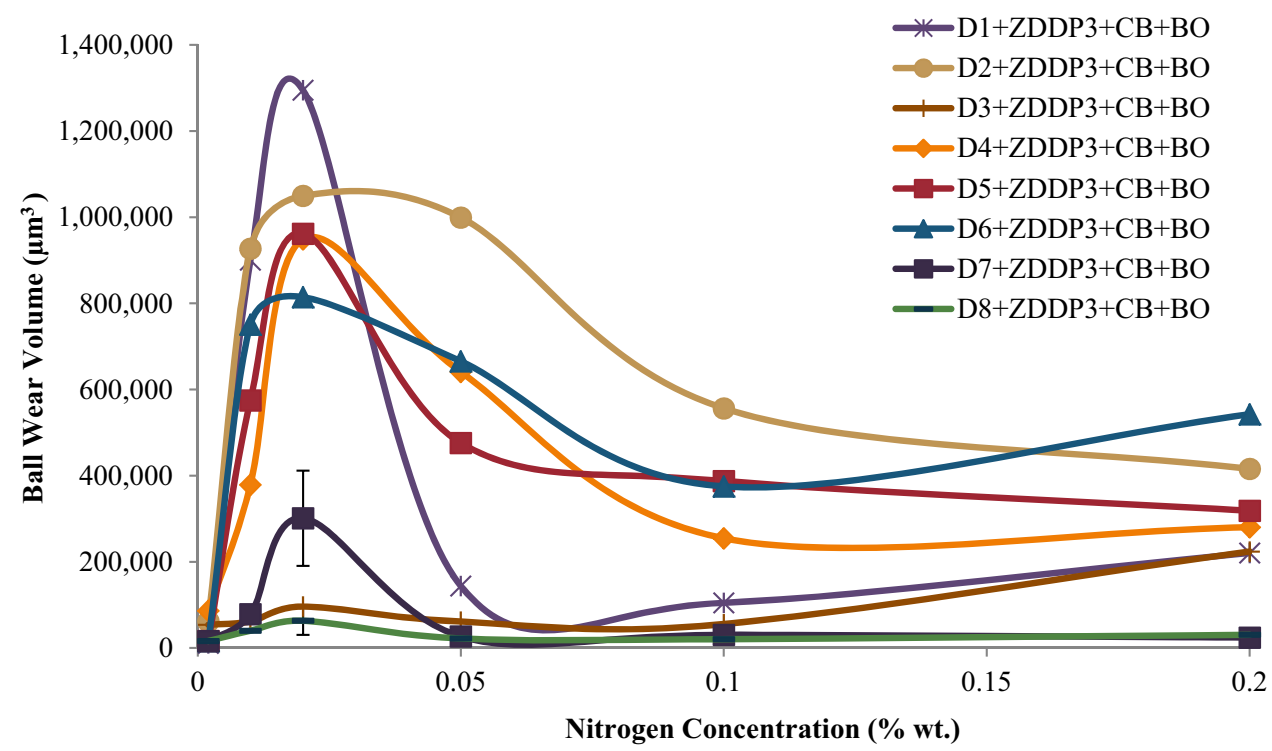




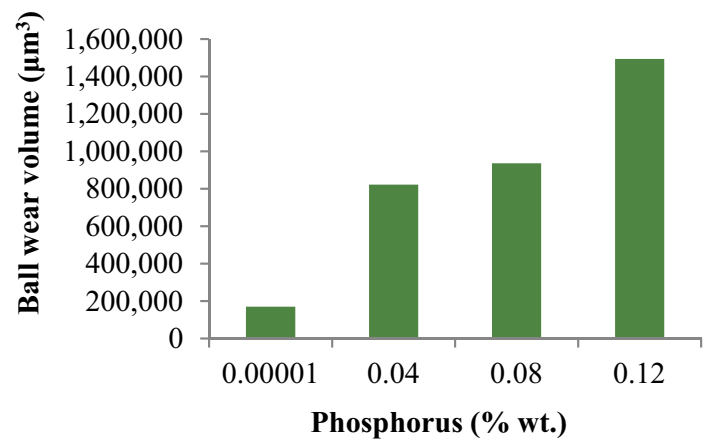

Fig. 4 HFRR ball wear volume of $\mathrm{D} 4+\mathrm{ZDDP} 3+\mathrm{CB}+\mathrm{BO}$ at increasing $\mathrm{P}$ concentration; ( $5 \mathrm{wt} \% \mathrm{CB})$

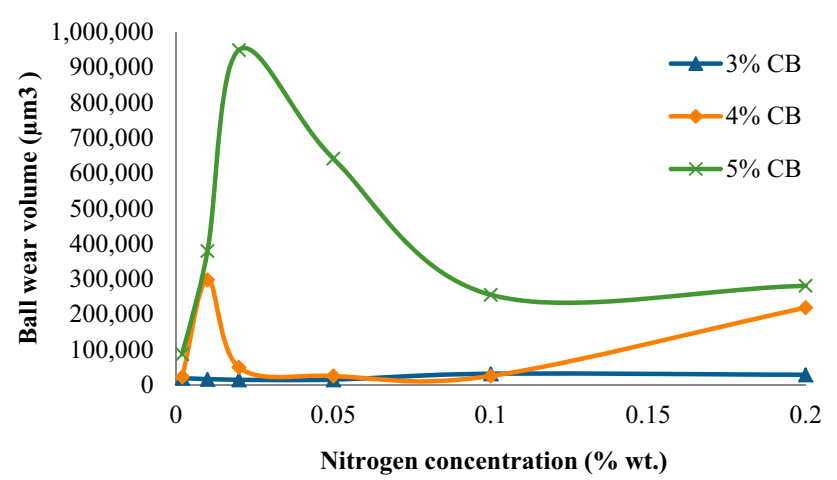

Fig. 5 HFRR ball wear volume of D4 + ZDDP3 + CB + BO at different CB concentrations; $(0.08$ wt $\%$ P)

\subsection{Effect of Phosphorous Concentration}

An oil blend that gives high wear rates at intermediate dispersant concentration (D4 + ZDDP3 + CB + BO) was chosen to be further investigated by varying the ZDDP concentration. Figure 4 shows how the ball wear volume varies with increasing phosphorus (ZDDP) level. Wear volume increases almost linearly with ZDDP concentration, supporting the idea that the reaction of ZDDP with the rubbing surfaces is a rate-determining step in the wear process.

\subsection{Effect of CB Concentration}

A similar investigation was carried out by varying the $\mathrm{CB}$ concentration. Figure 5 shows the ball wear volume losses at 3, 4 and $5 \mathrm{wt} \%$ of $\mathrm{CB}$ at varying nitrogen concentrations. The wear volume drops as the $\mathrm{CB}$ concentration decreases, reaching low values at $3 \mathrm{wt} \%$ of $\mathrm{CB}$. There is also a shift of the maximum of $5 \mathrm{wt} \% \mathrm{CB}$ from $0.02 \mathrm{wt} \% \mathrm{~N}$ to $0.01 \mathrm{wt} \%$ $\mathrm{N}$ at $4 \mathrm{wt} \%$ of $\mathrm{CB}$.

\section{Discussion and Ancillary Tests}

\subsection{Possible Origins of Wear Behaviour}

The above results show that the high wear seen with a combination of ZDDP and CB is very dependent on dispersant concentration and type. This clearly has practical significance and the reminder of this paper is concerned with investigating the origins of the response summarised in Fig. 3. Of primary interest are (a) why there is a maximum in wear at intermediate $\mathrm{N}$ concentration and (b) why some dispersants do not show this high wear but most do?

It is now generally accepted that $\mathrm{CB}$, being harder than most ZDDP films, abrades the tribofilm formed by ZDDP very rapidly and that the resulting loss of ferrous material is responsible for the high wear often seen when both ZDDP and $C B$ are present in a lubricant [19,21]. For this process to occur a number of conditions must be met:

- the CB particles must enter the rubbing contact;

- they need to adhere to and accumulate on the surface;

- the ZDDP must react with the rubbing surfaces;

- the CB particles must abrade the ZDDP tribofilm as fast as it forms.

The dispersant is likely to influence some or all of these processes. Thus via the effectiveness of CB dispersion it might control the entry of particles into the contact; via steric stabilisation it might limit CB particle adhesion to the rubbed surfaces; it might complex with ZDDP [29] and thus reduce the latter's rate of surface reaction; it might adsorb on the incipient ZDDP surface and protect it from abrasion. The existence of a wear maximum at an intermediate dispersant concentration also implies that for most dispersants there are two processes in operation as dispersant concentration is increased, one that promotes wear followed, at higher dispersant concentration, by one that reduces wear.

To explore the above possible effects, a number of ancillary experiments were carried out as described below. Two dispersants were chosen for study based on the HFRR wear tests; one that gives low wear over the whole range of $\mathrm{N}$ concentrations (D8), and one that gives high wear (D4) at intermediate and, to a lesser extent, at high concentration.

\subsection{Influence of Dispersant Concentration on Viscosity and Particle Size}

Figure 6 shows the dependence of viscosity on dispersant concentration and shear rate at low shear rates for the two dispersants blends with ZDDP and CB. At very low shear rates, viscosity decreases rapidly as dispersant concentration increases for both dispersant systems. The suspension 
Fig. 6 Viscosity versus shear rate results of two dispersant blends with ZDDP, CB and BO at various $\mathrm{N}$ concentrations in $\%$ wt.; (0.08 wt \% P, 5 wt \% CB). (Color figure online)
$\mathrm{D} 4+\mathrm{ZDDP} 3+\mathrm{CB}+\mathrm{BO}$

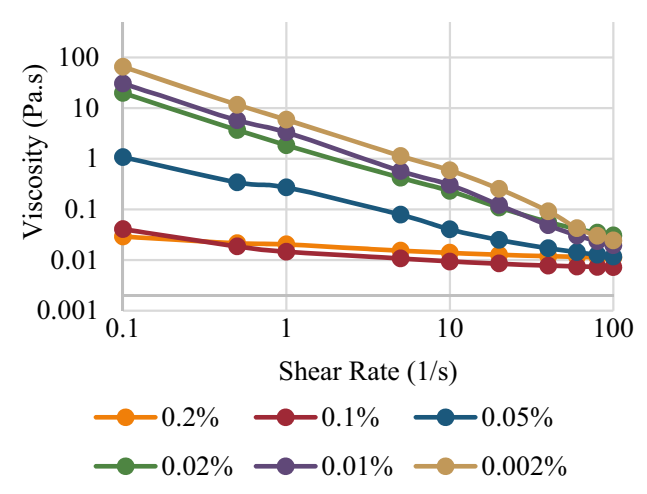

$\mathrm{D} 8+\mathrm{ZDDP} 3+\mathrm{CB}+\mathrm{BO}$

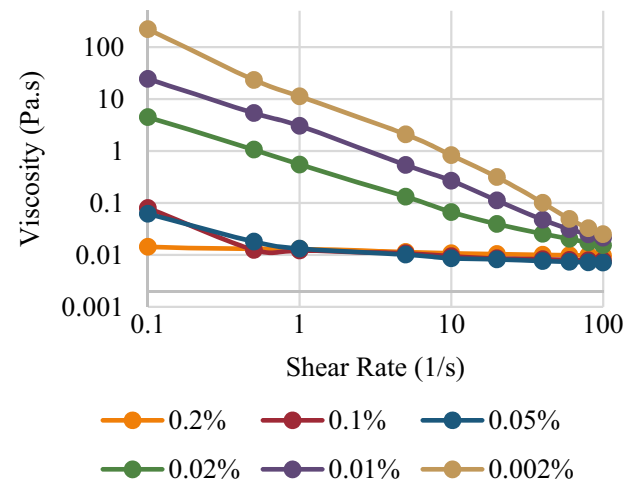

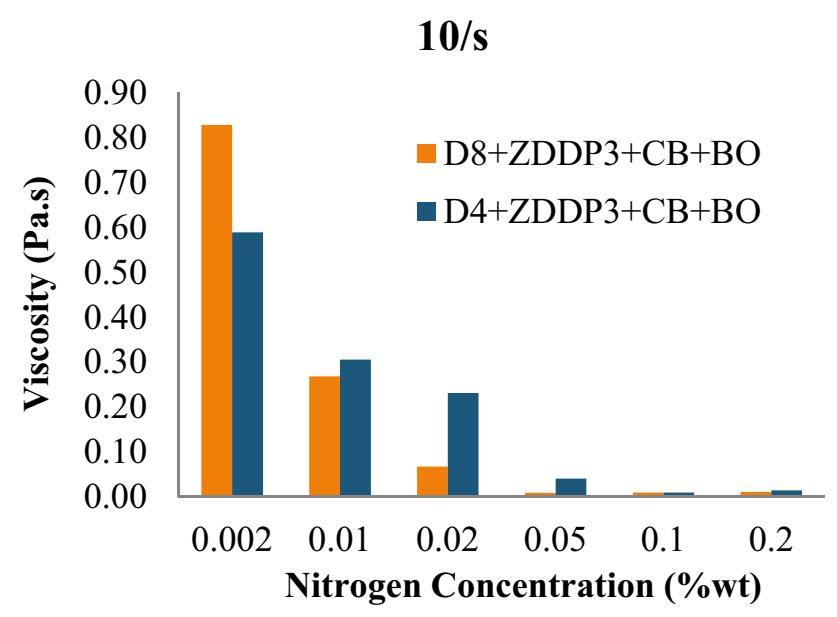

Fig. 7 Viscosity of D4 and D8 dispersant blends with ZDDP3, CB and $\mathrm{BO}$ at various $\mathrm{N}$ concentrations; $(0.08 \mathrm{wt} \% \mathrm{P}, 5 \mathrm{wt} \% \mathrm{CB})$. (Color figure online)

rheology also depends very strongly on shear rate at very low shear rates when the oils contain low dispersant concentrations $(0.002,0.01,0.02$ and $0.05 \mathrm{wt} \%$ of $\mathrm{N})$. Then, as the shear rate increases, all the oil blends become very similar in viscosity. This probably represents break down of loose aggregates of secondary particles (sometimes called tertiary structure), resulting in shear thinning. The low nitrogencontent D4 blends display non-Newtonian behaviour, with the viscosity rapidly decreasing with shear rate. In contrast, the blends at $0.2 \mathrm{wt} \% \mathrm{~N}$ and $0.1 \mathrm{wt} \% \mathrm{~N}$ show almost Newtonian characteristics with viscosity remaining quite constant with increasing shear rate. This is believed to be because the high $\mathrm{N}$ concentrations disperse the tertiary structures so well that either they are no longer present or they break down at extremely small shear rates. D8 in ZDDP + CB + BO, shows almost Newtonian behaviour at $0.05 \mathrm{wt} \% \mathrm{~N}$ while the $\mathrm{D} 4$ requires $0.1 \mathrm{wt} \% \mathrm{~N}$ to achieve this response.

Figure 7 shows the viscosity of each sample at $10 \mathrm{~s}^{-1}$ shear rate for all $\mathrm{N}$ concentrations. As $\mathrm{N}$ concentration

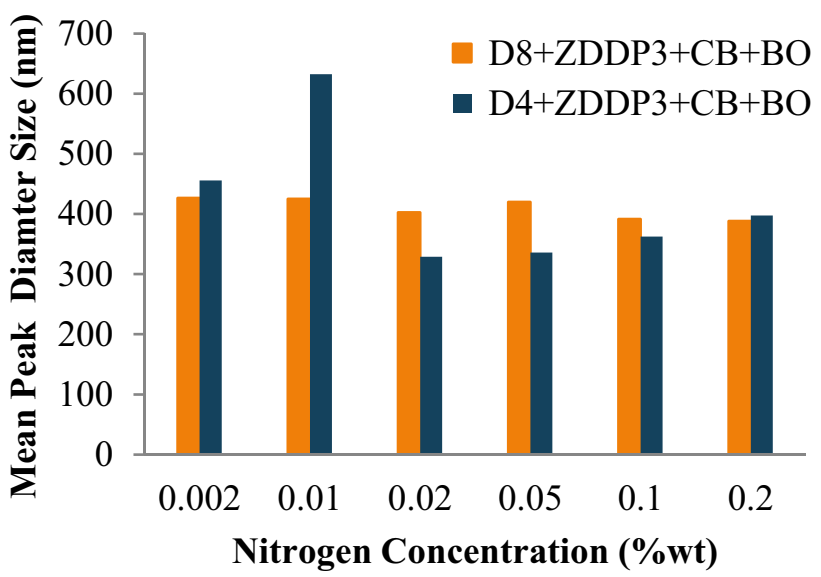

Fig. 8 DLS results of the mean peak aggregate size of D4 and D8 blends with ZDDP, $\mathrm{CB}$ and $\mathrm{BO}$ at various $\mathrm{N}$ concentrations; $(0.08$ wt \% P, 5 wt \% CB). (Color figure online)

increases, the viscosity decreases markedly for all the samples.

Figure 8 compares the mean particle size of CB dispersions measured using DLS for the two dispersants at various dispersant concentrations. As mentioned in Sect. 2 of this paper, the primary particle size of the CB used in this study ranges between 20 and $50 \mathrm{~nm}$. Similar values were also reported by Clague et al. [30], Lapuerta et al. [31] and La Rocca et al. [32]. These primary particles are fused strongly together to form secondary particle aggregates with average diameter size of 120-500 nm [27, 29, 32]. In the absence of effective dispersion these secondary aggregates can link together to form larger tertiary structures of up to $1 \mu \mathrm{m}$ [33]. According to the DLS results in Fig. 7, the mean peak diameter size found in the samples is around $400 \mathrm{~nm}$. This refers to the mean peak diameter size of the intensity distributions versus particle size and so represents the most frequent size found in the samples.

The DLS results show little evidence of agglomeration above $400 \mathrm{~nm}$ even at very low $\mathrm{N}$ concentrations except for 
D4 at $0.01 \mathrm{wt} \% \mathrm{~N}$. This contradicts the viscosity measurements that suggest considerable aggregation at a combination of low dispersant concentration and low shear rate. This discrepancy probably results from the heptane dilution procedure washing out the dispersant from the particles prior to DLS. This procedure was adopted from La Rocca et al. [34] who reported that this process puts little strain on the soot aggregates so that it should be expected to reveal a structure typical of the soot as it was in the engine oil [35]. However, there is no evidence that this is the case and the above results suggest that DLS is under-estimating the effective particle size in oil suspensions at low $\mathrm{N}$ concentrations. TEM is an alternative method of measuring size distributions but this also involves dilution in a medium and cleaning of the samples from oil. One possible way to minimise any change of aggregation due to the medium used might be to add dispersant to heptane but that was beyond the scope of this study and further investigation was not undertaken.

\subsection{Surface Adhesion via SEM-FEG}

One possible effect of dispersant is to control the extent to which $\mathrm{CB}$ particles enter the contact and adhere to the rubbing surfaces. This was explored by examining rubbed surfaces using SEM-FEG. Figure 9 shows images of the disc surface wear tracks at $100 \mathrm{k}$ magnification after testing with various concentrations of D4 and D8 in ZDDP solution with CB. The discs were rinsed lightly with heptane prior to imaging to remove most supernatant fluid. At a very low dispersant concentration of $0.002 \mathrm{wt} \% \mathrm{~N}$, very few particles appear to be present on the rubbed surfaces. The darker areas (stains) on the surfaces indicate the existence of a ZDDP film. At $0.02 \mathrm{wt} \% \mathrm{~N}$ there are many secondary $\mathrm{CB}$ particles on discs from both D4 and D8 tests. At higher N concentrations, there are very large aggregates at D4 surface. On the D8 surface at $0.1 \mathrm{wt} \% \mathrm{~N}$ there is some ZDDP film along the wear track with some particles lying on it. The particles are
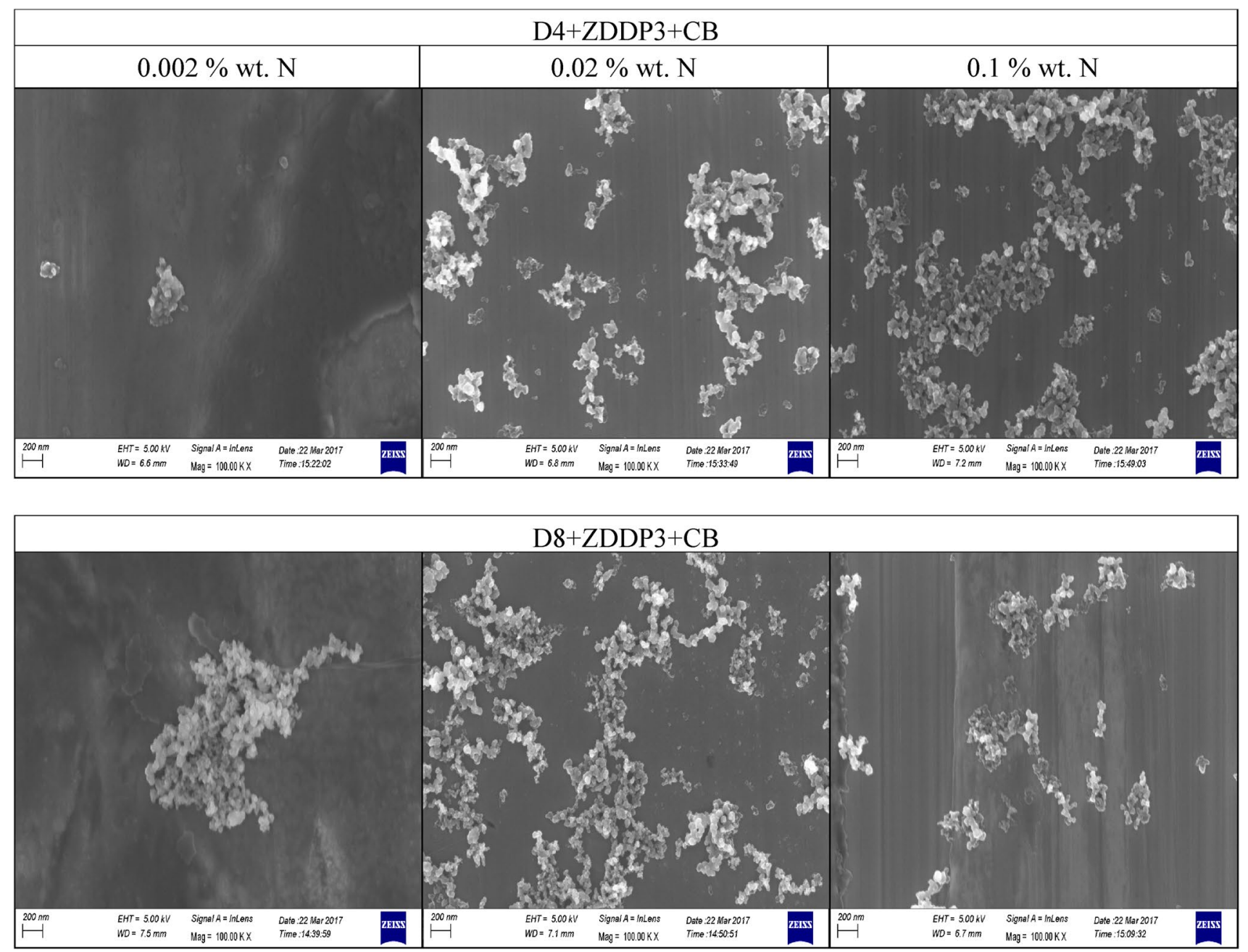

Fig. 9 SEM-FEG disc images of D4 and D8 containing solutions with CB at various N concentrations inside the wear track at $100 \mathrm{k}$ magnification and $200 \mathrm{~nm}$ scale; $(0.08 \mathrm{wt} \% \mathrm{P}, 5 \mathrm{wt} \% \mathrm{CB})$ 
Fig. 10 SEM-FEG disc image of D8 containing solution with $\mathrm{CB}$ at $0.02 \mathrm{wt} \% \mathrm{~N} ;(0.08 \mathrm{wt} \% \mathrm{P}$, $5 \mathrm{wt} \% \mathrm{CB})$

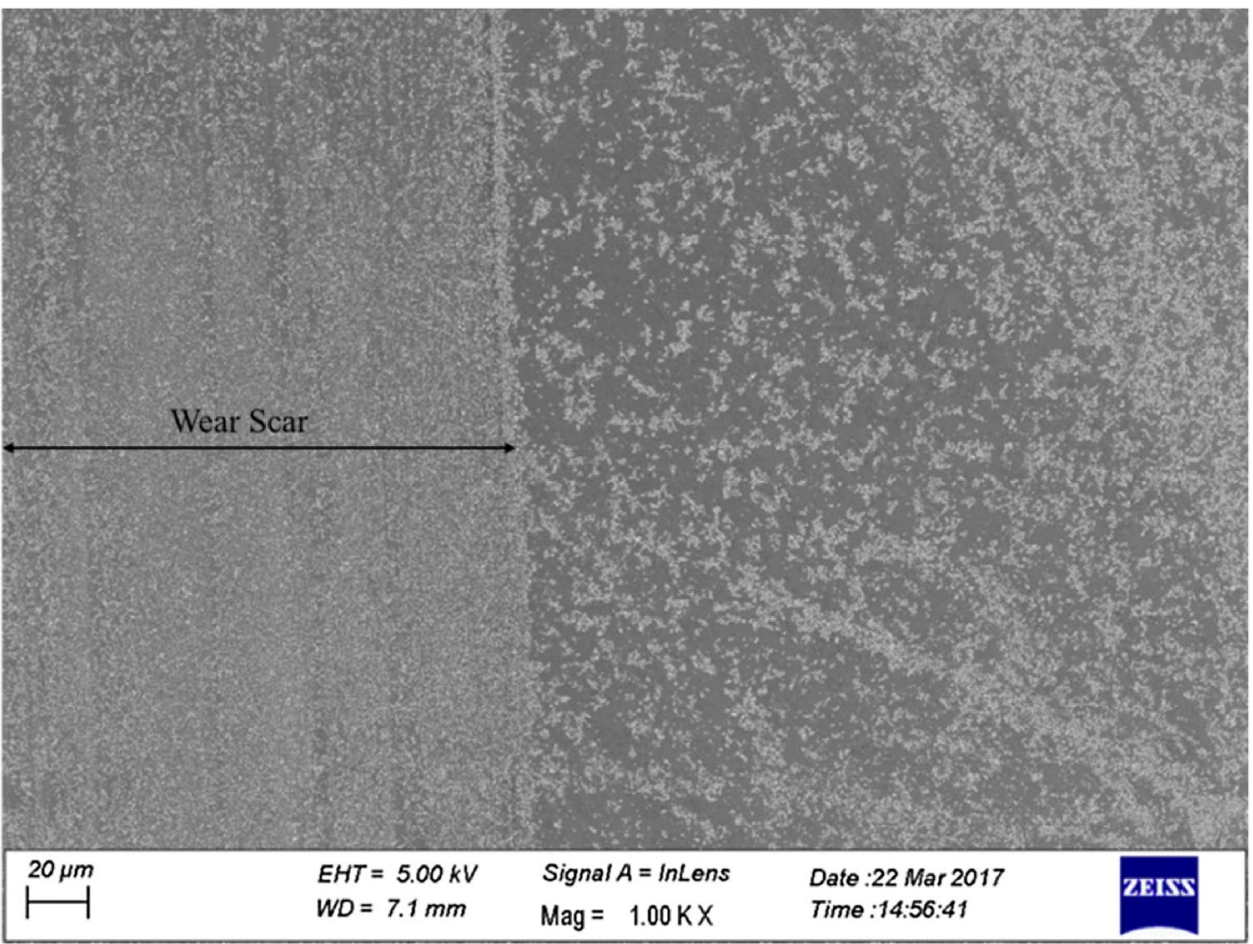

about $200 \mathrm{~nm}$ while the ones on D4 disc are significantly bigger, ranging from $100 \mathrm{~nm}$ to $1 \mu \mathrm{m}$.

Figure 10 shows an image that encompasses both inside and outside the rubbed track for D8 solution with $\mathrm{CB}$ at $0.02 \mathrm{wt} \% \mathrm{~N}$. It is interesting to observe how the CB particles have broken up in the contact to give many small particles inside, but fewer, bigger ones visible outside the wear track. However, this dispersion gives low wear in HFRR tests suggesting that the effectiveness of D8 in preventing CB wear at intermediate concentrations does not originate from it preventing adhesion of particles to the surface.

\subsection{Influence of Dispersant on ZDDP Reaction Rate}

One possible effect of dispersant might be to reduce the rate at which ZDDP reacts with surfaces in the rubbing contact. It is well known that ZDDP and dispersant molecules interact in solution to reduce the rate of ZDDP film build-up and to promote wear [29]. This is generally considered to be undesirable but if the ZDDP reaction promotes wear when $\mathrm{CB}$ is present then such interactions may actually reduce wear.

Figure 11 shows the influence of the two dispersants on ZDDP film formation rate at $0.02 \mathrm{wt} \% \mathrm{~N}$ concentration using MTM-SLIM. The first row shows film formation by ZDDP3 alone in solution while the image sequences in rows 2 and 4 show the impact of the two dispersants D4 and D8 on ZDDP film formation. With just ZDDP3, the tribofilm becomes very thick after $60 \mathrm{~min}$ of rubbing.
As noted by other researchers, the dispersants D4 and D8 both slow tribofilm formation markedly, D8 more than D4. Similar behaviour was seen at a dispersant concentration of $0.1 \mathrm{wt} \% \mathrm{~N}$, though in this case the rate of ZDDP film formation was even slower for both dispersants. Rows 3 and 5 show the effect of replacing the ZDDP + dispersant solution by a similar one but containing $5 \mathrm{wt} \% \mathrm{CB}$. The ZDDP tribofilm is removed almost entirely after just 2 min rubbing for both dispersants. After 60 min rubbing the shape of the interference images becomes elliptical because of wear of the ball, especially for the solution containing D4.

These results confirm the role of CB in removing ZDDP tribofilm, as noted by previous researchers $[19,26]$. It also appears to provide some support for the possibility that dispersant D8 may limit wear by slowing the rate of reaction of ZDDP, but further tests showed that in statistical terms D4 and D8 actually slowed ZDDP film formation to about the same extent.

It is possible that, while different dispersants might not change the overall ZDDP film formation rate, they might influence differently the initial kinetics of formation. To explore this, Figs. 12 and 13 show the distribution of zinc, phosphorus and sulphur on MTM ball surfaces using EDS after just $5 \mathrm{~s}$ of rubbing in the reciprocating pure sliding MTM, using solutions of ZDDP and two dispersant + ZDDP combinations. It can be seen that there is little difference in the amount of these elements on these surfaces due to dispersant; indeed more film appears to 


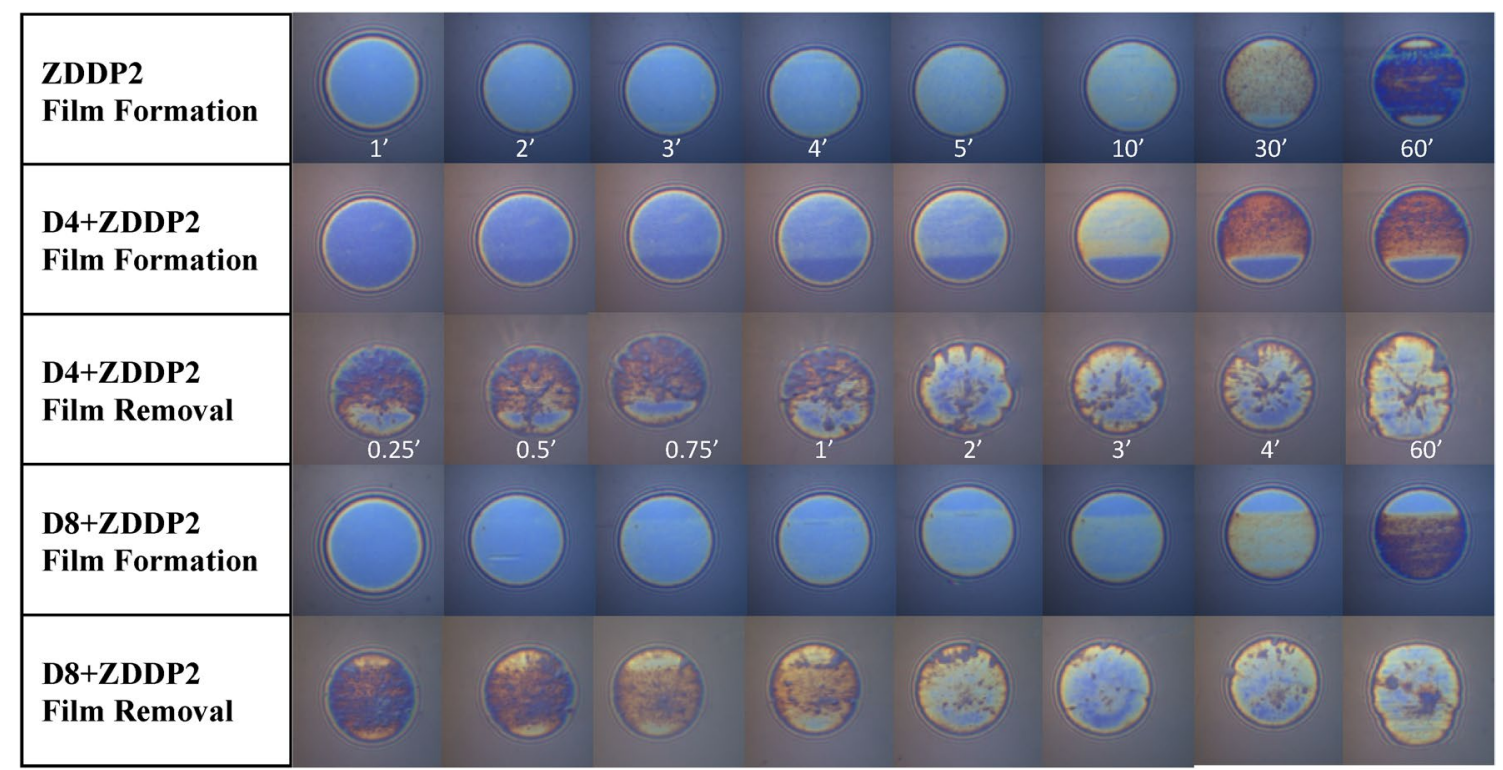

Fig. 11 MTM-SLIM film formation of D4 and D8 with ZDDP3 at 0.02\% wt. N and film removal by CB; (0.08 wt\% P, 5 wt\% CB). Rubbing times shown are in minutes

be present when $0.02 \mathrm{wt} \% \mathrm{~N}$ concentrations of dispersant is present that when none is present. However it can be seen that ZDDP film formation rate is much slower with $0.1 \mathrm{wt} \% \mathrm{~N}$ dispersant concentration. These results also confirm the rapid formation of sulphide in the film in pure sliding, reciprocating contact, as noted by Shimizu et al. [28].

\subsection{Origins of the Influence of Dispersant on ZDDP/ CB Wear}

Based on the viscosity and SEM-FEG measurements it appears likely that the increase in wear seen with most dispersants as dispersant concentration is increased from 0.002 $\mathrm{wt} \% \mathrm{~N}$ up to $0.02 \mathrm{wt} \% \mathrm{~N}$ originates primarily from the latters' effect of increasing the mobility of the CB particles so that they can enter the rubbing contact. This is evident from Fig. 9 where very few particles are seen on the rubbed surfaces at low $\mathrm{N}$ concentration. At very low $\mathrm{N}$ concentrations the $\mathrm{CB}$ dispersions are extremely viscous and almost paste-like due to interactions between secondary particles to form aggregate structures. Indeed a key role of dispersant additives is to prevent these structures.

From Figs. 9 and 10 it is clear that by $0.02 \% \mathrm{~N}$ concentration of dispersant, $\mathrm{CB}$ particles are entering the contact and accumulating on the rubbed surfaces. However this behaviour is not, of itself, sufficient to cause the high wear seen in Fig. 3, since such wear is not seen with D8 even though the surface has a high CB particle coverage. Also the rubbed surface continues to possess adhered CB particles even at high D4 concentrations when wear becomes relatively low.
Thus the beneficial effect on CB/ZDDP wear at high dispersant concentrations, or for a few dispersants at all concentrations, must originate from the effect of dispersant on either ZDDP reaction rate or ZDDP reaction film removal by CB.

The idea that high concentrations of dispersants may reduce the reaction rate of ZDDP and thus slow the corrosive-abrasive process is appealing since it is evident from Figs. 11 and 13 that high dispersant concentrations do indeed slow ZDDP reaction. It would also be consistent with the fact that those dispersants that are able to suppress high wear at intermediate concentrations are ones of high functionality, as indicated in Table 1 . Unfortunately direct comparison of such a dispersant, D8, with one that allows high wear, D4, suggests that both the initial ZDDP reaction rate with rubbed surfaces and its subsequent film growth are similar, even though the former shows very low and the latter high wear at intermediate concentrations.

The remaining possibility is that at high concentrations of dispersant, and with dispersant having high functionality and high MWt., at all concentrations, the dispersant effectively protects the incipient ZDDP tribofilm from wear, thus suppressing the loss of iron-containing compounds from the surface. This could be achieved either by it adsorbing on the reaction film and effectively acting as an anti-wear additive, or by adsorbing on the $\mathrm{CB}$ particles so strongly as to prevent them from damaging the tribofilm. In either case it is evident from Fig. 11 that the dispersant does not protect the whole ZDDP tribofilm, since this is removed rapidly when CB is added. Thus the dispersant must be acting to protect only the very thin, initial ferrous-based reaction film that is too thin to be detected by SLIM. 
Fig. 12 Film formation of D4 and $\mathrm{D} 8$ with ZDDP3 at 0.02 $\mathrm{wt} \% \mathrm{~N}$ after $5 \mathrm{~s}$ of rubbing in reciprocating MTM; $(0.08 \mathrm{wt} \%$ P)

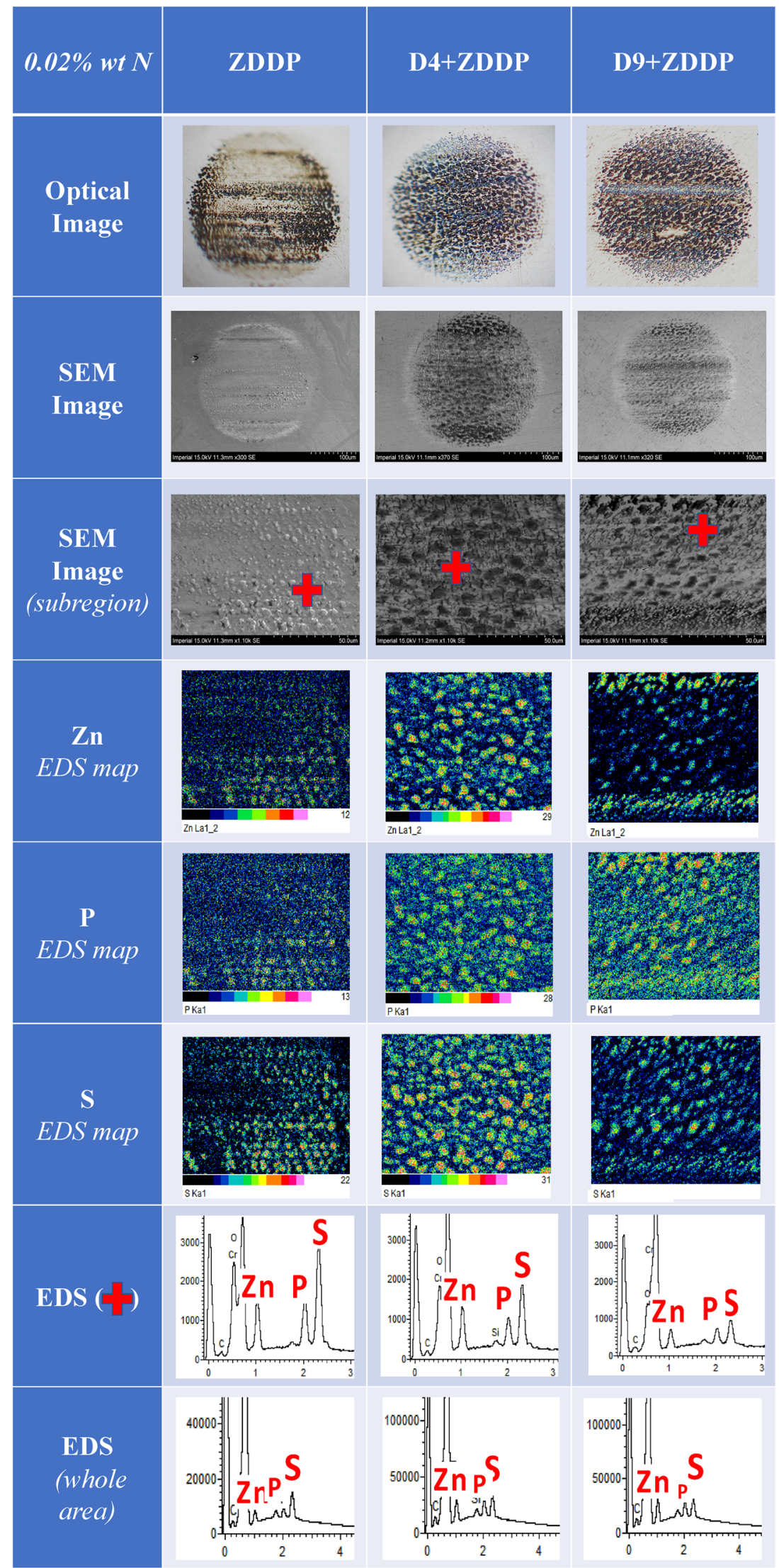


Fig. 13 Film formation of D4 and D8 with ZDDP3 at $0.1 \mathrm{wt} \%$ $\mathrm{N}$ after $5 \mathrm{~s}$ of rubbing in reciprocating MTM; $(0.08 \mathrm{wt} \% \mathrm{P})$

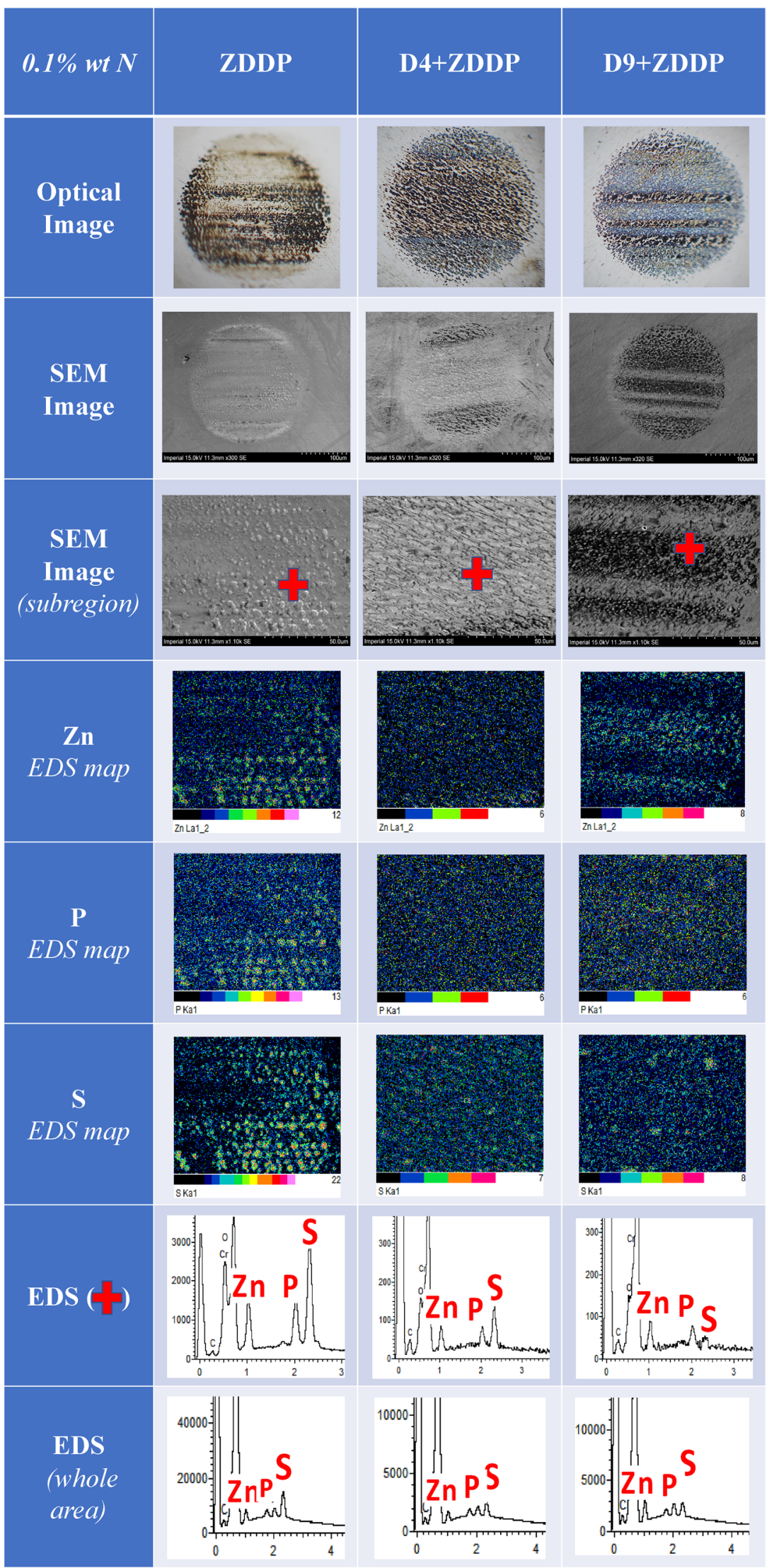




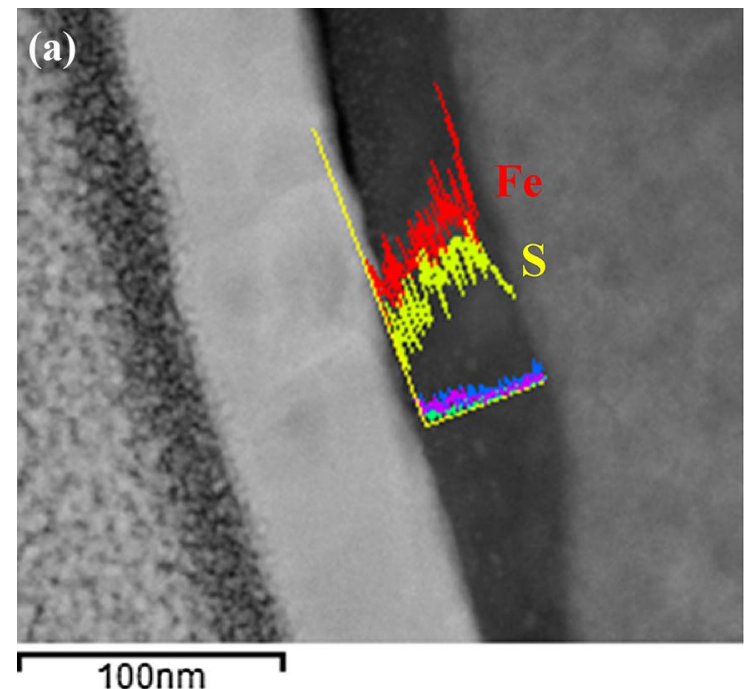

Fig. 14 a FIB-EDS section of the rubbed HFRR disc track from a test using $\mathrm{D} 8+\mathrm{ZDDP}+\mathrm{CB}$ at $0.02 \mathrm{wt} \% \mathrm{~N}$, showing the presence of a $60 \mathrm{~nm}$ iron sulphide film and no phosphorus-containing film; red

This was investigated using focussed ion beam milling with transmission electron microscopy on HFRR disc specimens. Scanning mode TEM (STEM) was applied in order to use energy-dispersive X-ray spectroscopy (EDX) to obtain elemental composition information. Figure 14a shows a TEM/EDS image of the cross section of the rubbed surface from a test using D8 + ZDDP + CB at $0.02 \mathrm{wt} \% \mathrm{~N}$. The steel substrate is on the right and to the left of this is a $c a 60 \mathrm{~nm}$ thick film that, as the EDS line analysis shows, consists almost entirely of $\mathrm{Fe}$ and $\mathrm{S}$. This is covered by a protective Au layer and then, to the left of the image a further protective Pt layer. As shown in Fig. 14b, high resolution TEM shows the film to be nanocrystalline with a domain size of up to $10 \mathrm{~nm}$. From repeat TEM measurements this film varied between 60 and $80 \mathrm{~nm}$ in thickness. With D4 a much thinner and very variable, $35 \mathrm{~nm}$ sulphur-rich film was found.

It must be emphasised that this is not definitive evidence that D8 protects the incipient ZDDP film more effectively than D4 since a single FIB-TEM analysis only looks at a very small region of the surface, so if the film is patchy it may yield unrepresentative results. Indeed analysis of a sample rubbed in $\mathrm{D} 7+\mathrm{ZDDP}+\mathrm{CB}$, which gave quite low HFRR wear at intermediate concentration showed no sulphide at all. Further work thus requires many FIB-TEM to be carried out to test whether there is a statistically significant correlation between

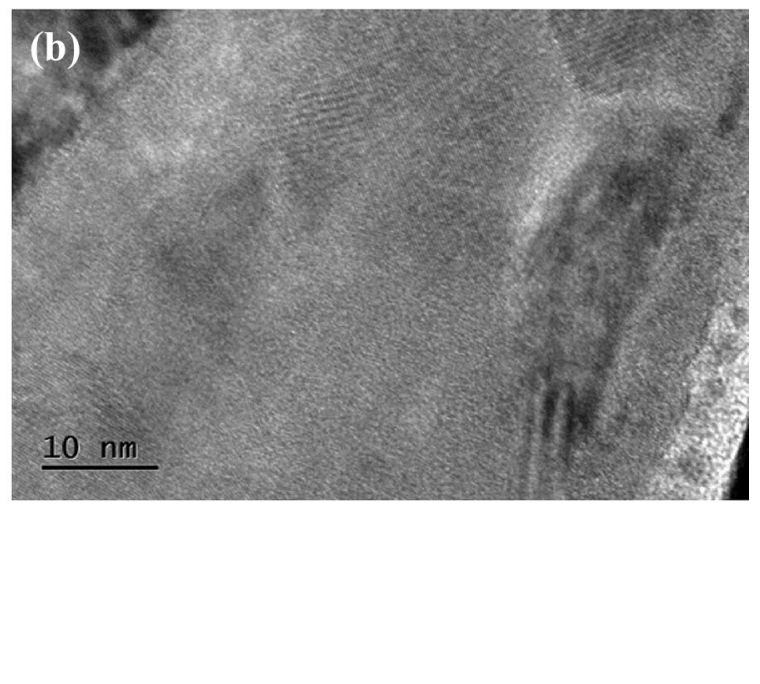

line is Fe and yellow is S. b FIB-TEM image of film showing partially nanocrystalline structure. $(0.08 \mathrm{wt} \% \mathrm{P}, 5 \mathrm{wt} \% \mathrm{CB})$. (Color figure online)

the incipient tribofilm thickness and the wear level for different dispersants.

It is noteworthy that this formation of a quite thick, homogeneous iron sulphide film is quite different from that normally seen with ZDDP, where there is generally a mixed S/P based film close to the steel substrate and a thick, primarily phosphate film on top of this. It appears that continual removal of the phosphate film by CB allows build-up of a thick sulphide film.

\section{Conclusions}

Previous work has shown that the engine oil anti-wear additive ZDDP can behave as a pro-wear rather than an anti-wear agent when soot or other solid nanoparticles are present. This is believed to originate from the soot abrading the ZDDP tribofilm as rapidly as it forms, resulting in a corrosive-abrasive wear mechanism. There is, however, another key additive present in engine oils, the dispersant, which is essential to maintain soot in suspension. This additive is designed to adsorb on soot particle surfaces and is also known to interact with ZDDP. It is thus of considerable interest to establish the role, if any, that this additive plays in soot wear. 
In the current study the influence of dispersant on soot wear with ZDDP has been studied using a range of different succinimide dispersants. Carbon black (CB) has been used as a soot surrogate. The most significant findings and conclusions from this study are summarised below:

- HFRR wear tests show that when ZDDP is added to solutions of dispersant and $\mathrm{CB}$, much more wear occurs than when ZDDP is left out. ZDDP thus has pro-wear properties in $\mathrm{CB}$ solutions, confirming previous studies.

- The rate of wear seen with ZDDP and CB increases with phosphorus concentration, suggesting that the ZDDP reaction with ferrous substrate is a rate-determining step in the wear process.

- Further HFRR tests show that both dispersant concentration and type strongly influence the wear induced by $\mathrm{CB}$ in ZDDP-containing oils. Most dispersants show a maximum of wear at $c a 0.02 \mathrm{wt} \% \mathrm{~N}$, which is around the concentration used in fully formulated engine oils. Much less wear is seen at very low and at high dispersant concentrations.

- A few dispersants do not show this high wear behaviour at intermediate concentrations and instead enable low wear over the whole concentration range studied. These appear to be succinimide dispersants combining high functionality and high MWt.

- The increase in wear with increasing dispersant concentration at very low dispersant concentrations is believed to originate from an increase in the ability of $\mathrm{CB}$ particles to enter the rubbing contact as they become better dispersed.

- Two possible origins of the lower wear seen at high dispersant concentrations than at intermediate concentrations for most dispersants have been identified. These are: (i) at high concentrations dispersants may interact with ZDDP molecules in solution or at surfaces to reduce the latters' reactivity and thus slow ZDDP reaction with rubbed surfaces; and (ii) at high concentrations dispersants may protect the initial ZDDP tribofilm from abrasion by adsorbing effectively either of the tribofilm itself or on the $\mathrm{CB}$ particles.

- The rates of ZDDP film formation by CB-free ZDDP blends containing dispersants that give high wear at intermediate dispersant concentrations are similar to those formed by blends containing dispersants that give low wear. This suggests that mechanism (i) above is not prevalent. Preliminary studies of the very thin ZDDP tribofilms present on rubbed surfaces when ZDDP and $\mathrm{CB}$ are present indicate that dispersants that enable low wear at intermediate concentrations are able to protect iron sulphide films on the rubbed surfaces, in support mechanism (ii) above.
- It is thus proposed that when ZDDP and soot are both present in an oil, the ZDDP reacts with the rubbed surfaces to form a film of iron sulphide that may be immediately abraded by the soot particles, resulting in rapid corrosive-abrasive wear. Key to this is that the initial tribofilm formed by ZDDP is iron sulphide and this is normally subsequently overlain by a protective zinc phosphate film [28]. However soot prevents build-up of this zinc phosphate film, thus allowing iron sulphide formation and removal to continue unchecked.

- High concentration levels of dispersant, and in particular some highly functionalised dispersants, appear to be able to protect this iron sulphide film from abrasion and so prevent the very high levels of wear otherwise seen with oils that contain both ZDDP and soot.

Acknowledgements This research was funded by Shell University Technology Centre (UTC) for Fuels and Lubricants.

Open Access This article is distributed under the terms of the Creative Commons Attribution 4.0 International License (http://creativeco mmons.org/licenses/by/4.0/), which permits unrestricted use, distribution, and reproduction in any medium, provided you give appropriate credit to the original author(s) and the source, provide a link to the Creative Commons license, and indicate if changes were made.

\section{References}

1. Li, S., Csontos, A.W., Gable, C.A., Jao, T.C.: Wear in Cummins M-11/EGR test engines. SAE Techn. Paper No. 2002-01-1672 (2002)

2. Soejima, M., Ejima, Y., Uemori, K., Kawasaki, M.: Studies on friction and wear characteristics of cam and follower: influences of soot contamination in engine oil. JSAE Rev. 23(1), 113-119 (2002)

3. Green, D.A., Lewis, R., Dwyer-Joyce, R.S.: Wear effects and mechanisms of soot-contaminated automotive lubricants. Proc. Inst. Mech. Eng., Part J: J. Eng. Tribol. 220(3), 159-169 (2006)

4. Rounds, F.G.: Carbon: cause of diesel engine wear. SAE Trans. 86, 2870-2881 (1977)

5. Rounds, F.: Effect of lubricant additives on the prowear characteristics of synthetic diesel soots. Lubr. Eng. 43, 273-282 (1987)

6. Berbezier, I., Martin, J., Kapsa, P.: The role of carbon in lubricated mild wear. Tribol. Int. 19(3), 115-122 (1986)

7. Hirose, Y.: Deterioration and wear characteristics of diesel-engine oil. J. Jpn. Soc. Lubr. Eng. 27(5), 394-395 (1982)

8. Corso, S., Adamo, R.: The effect of diesel soot on reactivity of oil additives and valve train materials. SAE Techn. Paper No. 841369 (1984)

9. Cusano, C., Sliney, H.: Dynamics of solid dispersions in oil during the lubrication of point contacts, part I-graphite. ASLE Trans. 25(2), 183-189 (1982)

10. Colacicco, P., Mazuyer, D.: The role of soot aggregation on the lubrication of diesel engines. Tribol. Trans. 38(4), 959-965 (1995) 
11. Yoshida, K.: Effects of sliding speed and temperature on tribological behavior with oils containing a polymer additive or soot. Tribol. Trans. 33(2), 221-228 (1990)

12. Yahagi, Y.: Corrosive wear of diesel engine cylinder bore. Tribol. Int. 20(6), 365-373 (1987)

13. Ryason, P., Chan, I., Gilmore, J.: Polishing wear by soot. Wear 137(1), 15-24 (1990)

14. Bardasz, E.A., Carrick, V.A., Ebeling, V.L., George, H.F., Graf, M.M., Kornbrekke, R.E., Pocinki, S.B.: Understanding soot mediated oil thickening through designed experimentation-Part 2: GM 6.5 L. SAE Techn. Paper No. 961915 (1996)

15. Nagai, I., Endo, H., Nakamura, H., Yano, H.: Soot and valve train wear in passenger car diesel engines. SAE Trans. 92, 986-1000 (1983)

16. Mainwaring, R.: Soot and wear in heavy duty diesel engines. SAE Techn. Paper No. 971631 (1997)

17. Van Dam, W., Willis, W.W., Cooper, M.W.: The impact of additive chemistry and lubricant rheology on wear in heavy duty diesel engines. SAE Techn. Paper No. 1999-01-3575 (1999)

18. Ratoi, M., Castle, R.C., Bovington, C.H., Spikes, H.A.: The influence of soot and dispersant on ZDDP film thickness and friction. Lubr. Sci. 17(1), 25-43 (2004)

19. Olomolehin, Y., Kapadia, R., Spikes, H.: Antagonistic interaction of antiwear additives and carbon black. Tribol. Lett. 37(1), 49-58 (2010)

20. Antusch, S., Dienwiebel, M., Nold, E., Albers, P., Spicher, U., Scherge, M.: On the tribochemical action of engine soot. Wear 269(1), 1-12 (2010)

21. Salehi, F.M., Khaemba, D.N., Morina, A., Neville, A.: Corrosiveabrasive wear induced by soot in boundary lubrication regime. Tribol. Lett. 63(2), 19 (2016)

22. Olomolehin, Y.: The influence of zinc dialkyldithiophosphate and other lubricant additives on soot-induced wear. PhD Thesis, Imperial College London (2010)

23. Stachowiak, G.W., Batchelor, A.W.: Engineering Tribology, Chap. 13, 3rd Edition publ. Butterworth Heinemann, (2006)

24. Booth, J.E., Nelson, K.D., Harvey, T.J., Wood, R.J.K., Wang, L., Powrie, H.E.G., Martinez, J.G.: The feasibility of using electrostatic monitoring to identify diesel lubricant additives and soot contamination interactions by factorial analysis. Tribol. Int. 39(12), 1564-1575 (2006)

25. Hu, E., Hu, X., Liu, T., Fang, L., Dearn, K.D., Xu, H.: The role of soot particles in the tribological behavior of engine lubricating oils. Wear 304(1-2), 152-161 (2013)

26. Kontou, A., Southby, M., Spikes, H.: Effect of steel hardness on soot wear. Wear 390, 236-245 (2017)

27. La Rocca, A., Di Liberto, G., Shayler, P.J., Parmenter, C.D.J., Fay, M.W.: Application of nanoparticle tracking analysis platform for the measurement of soot-in-oil agglomerates from automotive engines. Tribol. Int. 70, 142-147 (2014)

28. Shimizu, Y., Spikes, H.A.: The tribofilm formation of ZDDP under pure sliding, reciprocating conditions. Tribol. Lett. 64, 46 (2016)

29. Zhang, J., Yamaguchi, E., Spikes, H.: The antagonism between succinimide dispersants and a secondary zinc dialkyl dithiophosphate. Tribol. Trans. 57, 57-65 (2014)

30. Clague, A.D.H., Donnet, J.B., Wang, T.K., Peng, J.C.M.: A comparison of diesel engine soot with carbon black. Carbon 37(10), 1553-1565 (1999)

31. Lapuerta, M., Martos, F.J., Herreros, J.M.: Effect of engine operating conditions on the size of primary particles composing diesel soot agglomerates. J. Aerosol Sci. 38(4), 455-466 (2007)

32. La Rocca, A., Di Liberto, G., Shayler, P.J., Fay, M.W.: The nanostructure of soot-in-oil particles and agglomerates from an automotive diesel engine. Tribol. Int. 61, 80-87 (2013)

33. Sun, R., Kittelson, D.B., Blackshear, P.L.: Size distribution of diesel soot in the lubricating oil. SAE Trans. 100, 904-918 (1991)

34. La Rocca, A., Bonatesta, F., Fay, M.W., Campanella, F.: Characterisation of soot in oil from a gasoline direct injection engine using transmission electron microscopy. Tribol. Int. 86, 77-84 (2015)

35. Asango, A., La Rocca, A., Shayler, P.: Investigating the effect of carbon nanoparticles on the viscosity of lubricant oil from light duty automotive diesel engines. SAE Techn. Paper No. 201401-1481 (2014) 